\title{
Supporting Information \\ Tuning Catalytic Activity in Ultrathin Bimetallic \\ Nanowires via Surface Segregation: Some Insights
}

Shwetha Shettya, Meghabarna Gayen ${ }^{a}$, Sakshi Agarwal', Dipanwita Chatterjec ${ }^{a}$, Abhishek

Singh ${ }^{a}$, and N Ravishankar ${ }^{a *}$

*Email: nravi@iisc.ac.in

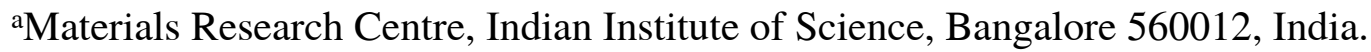

\section{Experimental Methods:}

\section{Chemicals:}

All the chemicals are used as received from the manufacturer. Gold (III) chloride $\left(\mathrm{HAuCl}_{4} \cdot \mathrm{XH}_{2} \mathrm{O}\right.$, Kem Light Laboratories Pvt. Ltd), Hexachloropalladate (IV) $\left(\mathrm{K}_{2} \mathrm{PdCl}_{6}\right.$, Sigma-Aldrich), Ethylene glycol (AR grade, SDFCL), D-Glucose extra-pure (AR grade, Sisco Research Laboratories Pvt. Ltd.), Oleylamine (> 97\% purity, Sigma-Aldrich), Triisopropylsilane (TIPS, Sigma-Aldrich), Hexane (AR grade, SDFCL).

\section{Au nanowire synthesis:}

The procedure is adapted from literature with minor modifications as per the requirement ${ }^{1}$. To synthesize the $\mathrm{Au}$ nanowires template, 22.8 umoles of $\mathrm{HAuCl}_{4}$ solution is mixed with $9 \mathrm{~mL}$ of 
hexane solvent and $300 \mu \mathrm{L}$ of oleylamine in a $15 \mathrm{~mL}$ centrifuge tube. This mixture is sonicated at room temperature for the complete dissolution of the gold precursor. To this golden color mixture, $450 \mu \mathrm{L}$ of TIPS is added and shaken well. The whole reaction mixture is then incubated at room temperature for 24 hours. The golden color turns to wine red once the Au nanowires start forming. Finally, the reaction mixture is centrifuged at $3000 \mathrm{rpm}$ for $3 \mathrm{~min}$ for to collect the nanowires. The washing procedure is repeated once again with hexane. The black product is redispersed in $2 \mathrm{~mL}$ hexane. To this $200 \mu \mathrm{L}$ of oleylamine is added to protect the nanowires from breaking.

AuPd nanowire synthesis: The method to synthesize AuPd nanowires is as described in the literature from our group ${ }^{2}$. The composition is varied by varying the Pd precursor concentration. To unambiguously identify AuPd nanowire samples with different Pd amount, we have named the samples as $\mathrm{Au}_{100-\mathrm{X}} \mathrm{Pd}_{\mathrm{X}}$ where $\mathrm{X}$ refers to the atom percentage of $\mathrm{Pd}$ mixed with $\mathrm{Au}$ as measured from wavelength dispersive X-ray spectroscopy (WDS) studies. Table 1 gives the molar ratios for Au and Pd precursors used to synthesize the compositionally tuned AuPd nanowires. The required molar amount of $\mathrm{K}_{2} \mathrm{PdCl}_{4}$ salt is added to $4 \mathrm{~mL}$ of ethylene glycol along with $200 \mu \mathrm{L}$ of oleylamine in a $10 \mathrm{~mL}$ microwave vial and sonicated to dissolve the Pd salt. Once this is done, $15.2 \mathrm{mM}$ of glucose is added and sonicated to dissolve it. The reaction mixture is kept undisturbed for the nonpolar pale yellow colored Pd-amine complex to form on top of polar EG solvent. To this bilayer mixture, as prepared Au nanowire solution is added dropwise so as not to disturb the layers. The tri-layer solution is then microwave heated at $150{ }^{\circ} \mathrm{C}$ for 1 hour. The black-colored top hexaneoleylamine layer is carefully transferred to a centrifuge vial and further washed sequentially with hexane, 1:1 mixture of hexane/ethanol and then with ethanol. During each step of washing, the 
sample is separated by centrifugation at an rpm of 3000 for 4 min. Finally, the samples are washed with hexane and kept in hexane for further use.

Table.S1: Molar ratios of Au and Pd precursors for the synthesis of compositionally tuned AuPd nanowires

\begin{tabular}{|c|c|c|}
\hline \multirow{2}{*}{ Sample } & \multicolumn{2}{|c|}{ Molar Ratio } \\
\cline { 2 - 3 } & $\mathrm{Au}$ & $\mathrm{Pd}$ \\
\hline $\mathrm{Au}_{85} \mathrm{Pd}_{15}$ & 1 & 0.1 \\
\hline $\mathrm{Au}_{75} \mathrm{Pd}_{25}$ & 1 & 0.22 \\
\hline $\mathrm{Au}_{60} \mathrm{Pd}_{40}$ & 1 & 0.44 \\
\hline $\mathrm{Au}_{50} \mathrm{Pd}_{50}$ & 1 & 0.66 \\
\hline $\mathrm{Au}_{25} \mathrm{Pd}_{75}$ & 1 & 1 \\
\hline
\end{tabular}

\section{Annealing AuPd nanowires in $\mathrm{CO}$ atmosphere:}

AuPd nanowires dispersed in hexane medium is drop-cast onto a glass slide or a carboncoated copper grid (for TEM studies). The sample is then transferred to a quartz boat and placed inside a tube furnace connected to a rotary vacuum pump. Temperature is ramped at a rate to 3 ${ }^{\circ} \mathrm{C} / \mathrm{min}$ to reach the target temperature of $170^{\circ} \mathrm{C}$. During the initial ramping vacuum is created. Once the temperature reaches $50{ }^{\circ} \mathrm{C}$, a gas mixture of $10 \% \mathrm{CO}$ (in nitrogen) is introduced for a brief period of $10 \mathrm{~min}$. The furnace is evacuated again. Such purging steps are performed at temperatures $80^{\circ} \mathrm{C}$ and $110^{\circ} \mathrm{C}$. Soon after this $\mathrm{CO}$ is filled till a pressure of $0.5 \mathrm{~atm} . \mathrm{CO}$ annealing 
is performed for a time of $90 \mathrm{~min}$ after which furnace is cooled to room temperature in $\mathrm{CO}$ atmosphere. The sample is re-dispersed in hexane for further use.

\section{CO-temperature programmed desorption (CO-TPD) studies}

CO-TPD studies are performed in Quantachrome Autosorb TPX iQ instrument attached to high purity (99.999\%) gas mixture of 10\% CO in nitrogen. Freshly prepared AuPd nanowires in hexane from a single batch are mixed with $500 \mathrm{mg}$ of fine silica beads using a mortar and pestle till a homogeneous solid mixture is formed. The powder is then dried in an oven at $70{ }^{\circ} \mathrm{C}$ for 2 hours. The solid black powder is then packed between quartz wool plugs in a chemisorption tube provided with the instrument. The sample is degassed under the continuous flow of helium for 30 min at $100{ }^{\circ} \mathrm{C}$. $\mathrm{CO}$ is adsorbed onto the sample at $35^{\circ} \mathrm{C}$ for one hour at a flow rate of $80 \mathrm{~mL} / \mathrm{min}$ followed by He gas purging for $30 \mathrm{~min}$. Temperature is ramped at $10{ }^{\circ} \mathrm{C} / \mathrm{min}$ till $250{ }^{\circ} \mathrm{C}$ under a continuous flow of $\mathrm{He}$ and the $\mathrm{CO}$ desorbed is measured using thermal conductivity detector.

\section{Electrochemical Measurements:}

The electrochemical activity of as-synthesized and CO-annealed AuPd nanowires are acquired using a conventional 3 electrode electrochemical set up with platinum foil as a counter electrode, $\mathrm{Ag} / \mathrm{AgCl}$ (saturated $\mathrm{KCl}$ ) as reference electrode connected to a potentiostat $(\mathrm{CH}$ Instruments). The working electrode is prepared by drop-casting the catalyst ink on to a Toray carbon paper strip of the fixed geometrical area of $0.5 \mathrm{~cm}^{2}$. The catalyst ink is made by dispersing freshly prepared AuPd nanowires in a solvent mixture of hexane/ethanol/Nafion $(5 \% \mathrm{w} / \mathrm{w})$ in 1:0.05:0.01 volume ratio. A homogeneous dispersion of catalyst ink is achieved by sonication and 
then coated on to the working electrode. Commercial Pd/C (10\%) electrode is prepared in a similar manner with $3 \mathrm{mg}$ of the dry catalyst powder dissolved in ethanol/Nafion $(5 \% \mathrm{w} / \mathrm{w})$ in 1:0.02 volume ratio.

Cyclic voltammetry scans for electrochemical surface area (ECSA) measurements are performed in an Ar purged 0.5 M KOH solution with a scan rate of $40 \mathrm{mV} / \mathrm{S}$ between the sweeping potentials of $-1.2 \mathrm{~V}$ to $0.7 \mathrm{~V}$ w.r.t. $\mathrm{Ag} / \mathrm{AgCl}$ electrode. The initial potential cycling is performed for 25 cycles for all the samples and the final $25^{\text {th }}$ potential cycle is used to evaluate the ECSA values. All the samples show well defined current waves for $\mathrm{H}_{2}$ desorption, $\mathrm{Pd}-\mathrm{O}$ and $\mathrm{Au}-\mathrm{O}$ reduction at potential values specific to each. The cathodic $\mathrm{Pd}-\mathrm{O}$ reduction wave is used to measure the ECSA for all the samples. By integrating the area under the Pd-O reduction wave, the total coulombic charge exchanged with the electrolyte is estimated ( $\mathrm{Q}_{\mathrm{Pd}-\mathrm{O}}$ in $\left.\mathrm{mAS}\right)$. ECSA is measured by dividing $\mathrm{Q}_{\mathrm{Pd}-\mathrm{O}}$ with $0.420 \mathrm{mC} / \mathrm{cm}^{2}$ which is the standard coulombic charge exchanged due to the reduction of the single oxide layer on a pure Pd surface. Specific ECSA with respect to the mass of the active metal $\mathrm{Pd}$ is calculated using the formula $\mathrm{Q}_{\mathrm{Pd}-\mathrm{O}} / 0.42 \times \mathrm{M}_{\mathrm{Pd}}$ where $\mathrm{M}_{\mathrm{Pd}}$ is the loading of the active metal Pd in AuPd or $\mathrm{Pd} / \mathrm{C}$ (in $\mathrm{mg} / \mathrm{cm}^{2}$ ).

\section{Characterization:}

Powder X-ray diffraction patterns were recorded using a PANalytical x'pert pro XRD system using $\mathrm{Cu}-\mathrm{Ka}(1.54060 \AA$ A $)$ radiation generated at $30 \mathrm{~mA}$ and $40 \mathrm{kV}$. Step size is $0.01^{\circ}(2 \mathrm{Th})$ per sec. Xpert High Score Plus software is used for the data analysis. The values of $2 \theta$ and lattice parameter for bulk $\mathrm{Au}$ and $\mathrm{Pd}$ are taken from International Centre for Diffraction Data (ICDD) powder diffraction file (PDF) numbers 00-004-0784 (for Au) and 00-001-1310 (for Pd). Scanning electron 
microscopy (SEM) images were acquired using Zeiss Ultra 55 Gemini field emission SEM. Brightfield and high-resolution transmission electron microscopy (BF- and HR-TEM) studies were done in Tecnai T20 TEM operated at $200 \mathrm{kV}$. The sample is drop-cast on a carbon-coated copper grid and dried under vacuum prior to analysis. X-ray photoelectron spectroscopy (XPS) measurements were recorded in Kratos Analytical Axis Ultra XPS instrument. Al Ka (1486.6 eV) monochromatic source is used for excitation. Samples are drop-cast on a cleaned Si wafer. High-resolution corelevel spectra of individual elements were acquired at a dwell time of $600 \mathrm{~ms}$ with a number of sweeps fixed at 9. Charge correction was done with respect to $\mathrm{C} 1 \mathrm{~s}$ at $284.6 \mathrm{eV}$. Data were analyzed using XPSPEAK4.1 software. The constraints for peak area ratios $\left(\mathrm{f}_{7 / 2}: \mathrm{f}_{5 / 2}=4: 3\right.$ and $\mathrm{d}_{5 / 2}: \mathrm{d}_{3 / 2}=$ 3:2), doublet separation ( $\mathrm{Au} 4 \mathrm{f}=3.7 \mathrm{eV}$ and $\mathrm{Pd} 3 \mathrm{~d}=5.3 \mathrm{eV})$, full width at half maxima (FWHM), are set using NIST XPS database values. Shirley background subtraction is used and peaks are fitted with Gauss-Lorentz sum functions. Samples are drop-cast on cleaned Si wafers to get a uniform film covering the entire area. All the data are acquired at room temperature. Temperature programmed desorption (TPD) studies are performed on Quantachrome Autosorb TPX iQ instrument which is connected to high purity $\mathrm{CO}$ gas mixture $\left(10 \% \mathrm{CO}+90 \% \mathrm{~N}_{2}\right)$ from Chemix gases. Atomic resolution STEM images were acquired in an aberration-corrected FEI Titan G2 80300 microscope, operated at $300 \mathrm{kV}$, spot size 10, beam current not more than $70 \mathrm{pA}$. Dwell time for the acquisition was $30 \mu$ sec. STEM-EDS map acquisition was done at $300 \mathrm{kV}$, spot size 10 , with beam current, maintained between $350-400 \mathrm{pA}$, dwell time of $800 \mu \mathrm{sec}$ and a total acquisition time of about 5 mins. For quantitative analysis, L-lines of Au and Pd were considered. WDS studies for the estimation of elemental composition is performed in Jeol-JXA8230 electron 
microprobe analyzer with tungsten source. The beam energy and current are fixed at $15 \mathrm{kV}$ and 50 nA.

a

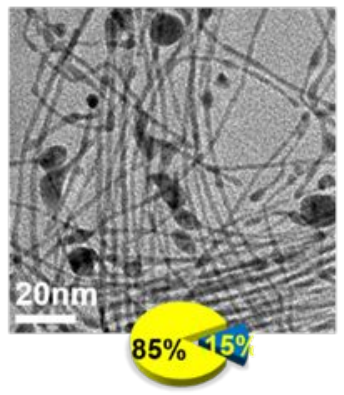

d

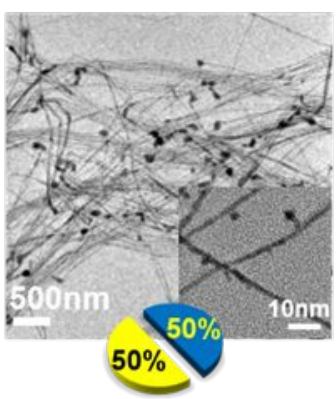

$\mathrm{b}$

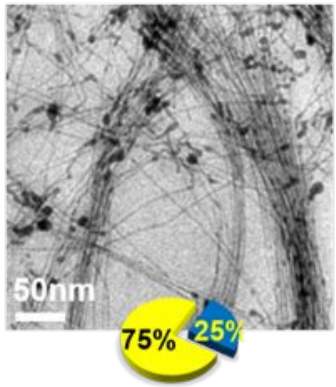

e

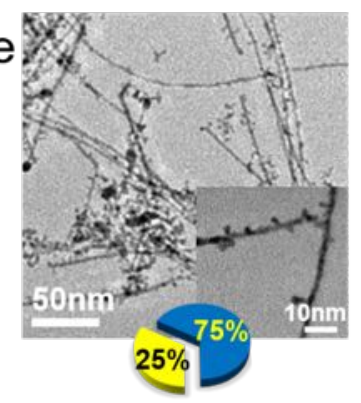

Au
C
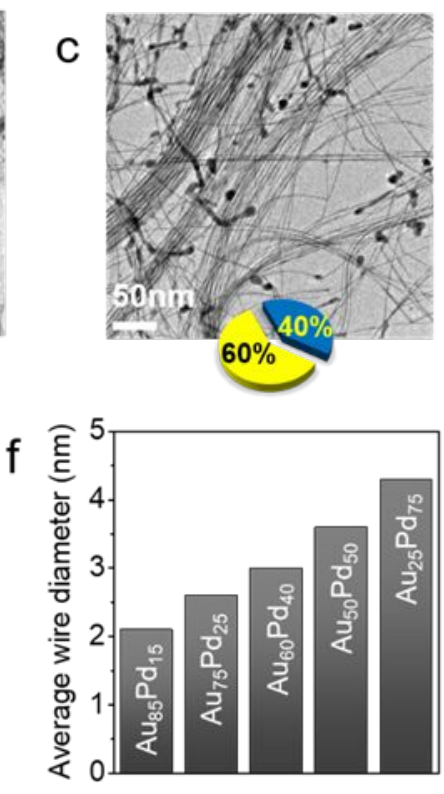
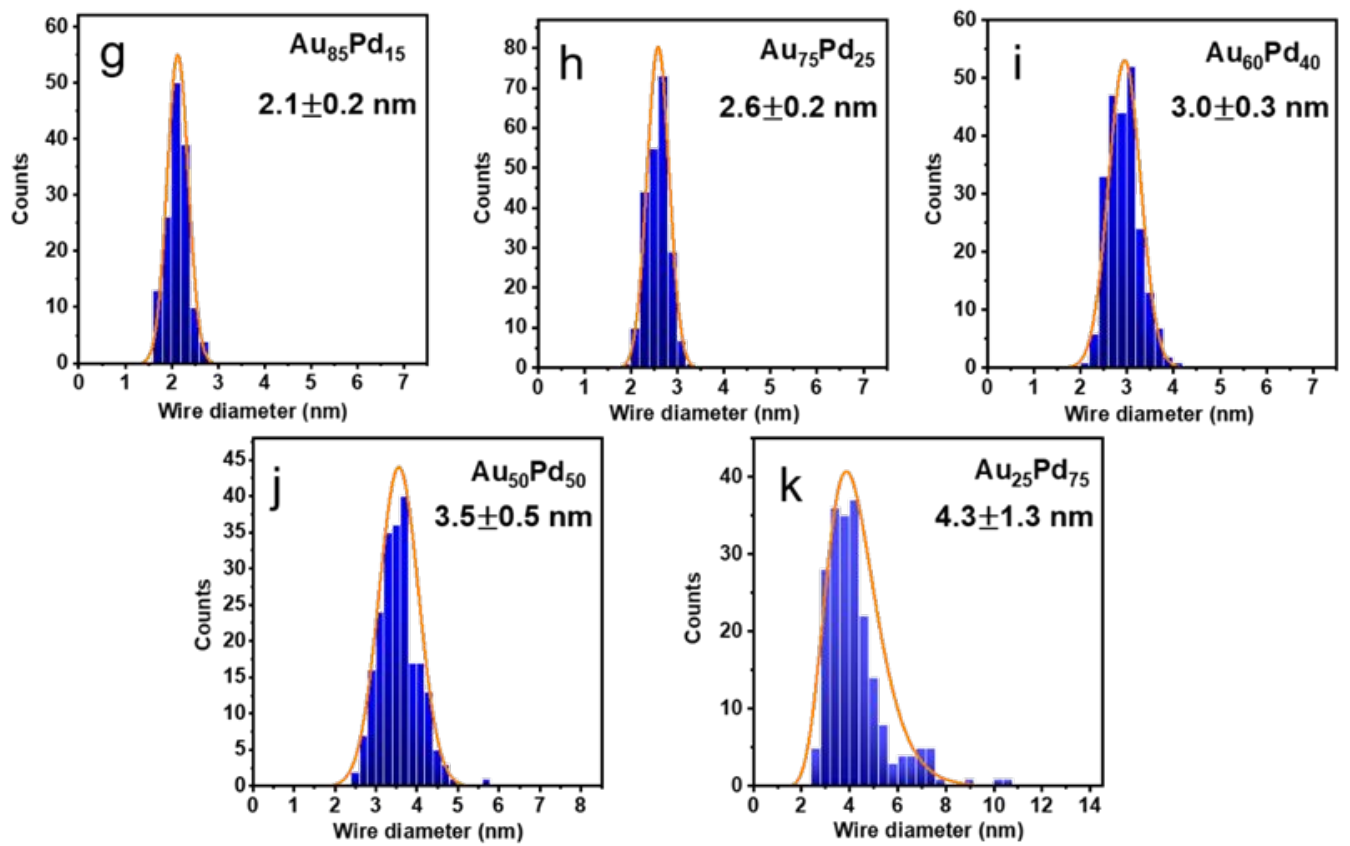
Figure S1: (a)-(e) Low magnification BF-TEM images of AuPd nanowires of varying atomic percentage of $\mathrm{Pd}$ (as shown in pi-chart) as measured from WDS studies. (f) Variation in average wire diameter with increase in atomic percent of Pd in AuPd nanowires. (g)-(k) Histogram of wire diameter for AuPd nanowires with varying composition. Mean diameter value is mentioned in each histogram plot. All the compositions show normal distribution except for $\mathrm{Au}_{25} \mathrm{Pd}_{75}$ which shows log normal distribution of wire diameter.
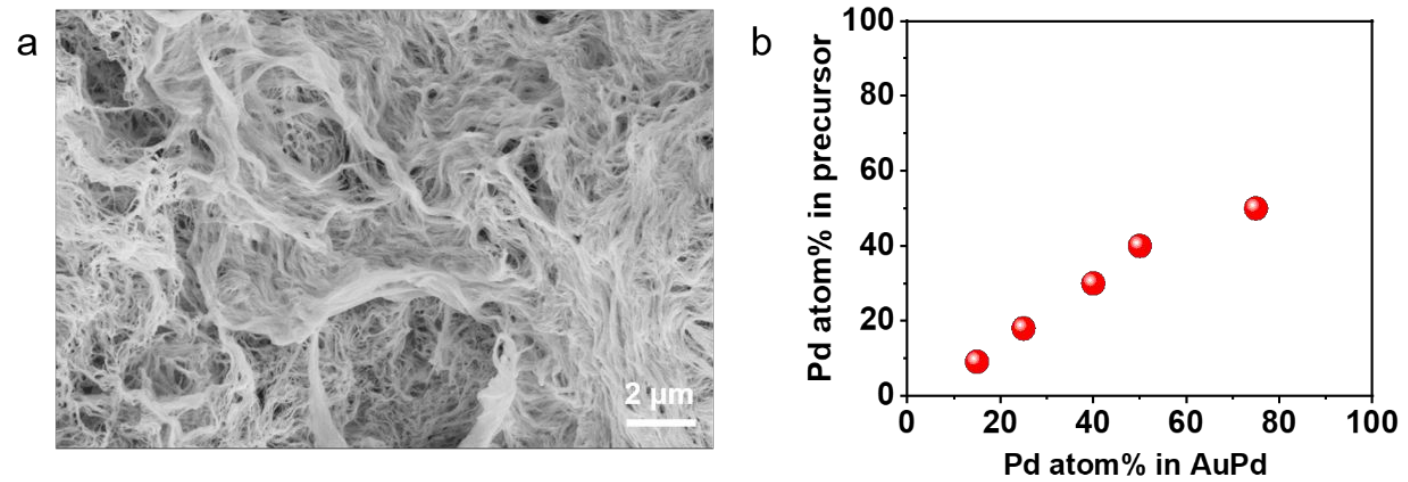

Figure S2: (a) Representative SEM image from AuPd nanowires depicting the high aspect ratio of the nanowires. (b) The plot of $\mathrm{Pd}$ atomic percentage from the molar concentration of $\mathrm{K}_{2} \mathrm{PdCl}_{6}$ precursor used for the synthesis of AuPd nanowires with respect of Pd atomic percentage from the WDS measurements of AuPd nanowires.

XRD Measurements:

Powder X-ray diffraction data for compositionally tuned AuPd nanowires shows that the nanowires preserve the FCC crystal lattice (Figure S3). The template Au nanowires conform to 
FCC crystal structure with (hkl) planes (111), (200), (220) and (311) at $2 \theta$ values at $37.9^{\circ}, 44.0^{\circ}$, $64.7^{\circ}$ and $77.6^{\circ}$ respectively4. For the AuPd nanowires the $2 \theta$ values of the prominent (111) peak lie in between that of Au nanowires and bulk Pd $\left(40.1^{\circ}\right)$ indicating the incorporation of $\mathrm{Pd}$ in $\mathrm{Au}$ and the alloying. The peaks broaden with the increase in Pd content which indicate the strain in the lattice as well as defects introduced when Pd is incorporated in the Au nanowire as reported earlier4 (Figure S4a). The lattice parameter 'a' for each composition is calculated from the (111) peak and plotted against the atomic fraction of Pd (Figure S4b). The lattice parameter variation with composition follows a pseudo-linear behavior and is lower than the value predicted using Vegard's law for the corresponding composition with bulk lattice parameter values for Au (4.08 $\AA)$ and Pd (3.89 ̊) indicating that not all the Pd may be incorporated in the solid solution form. This is in accordance with observation made by synchrotron XRD studies on compositionally tuned AuPd nanoicosahedra where it was observed that Pd amount in the core increases slower than the shell ${ }^{36}$. 


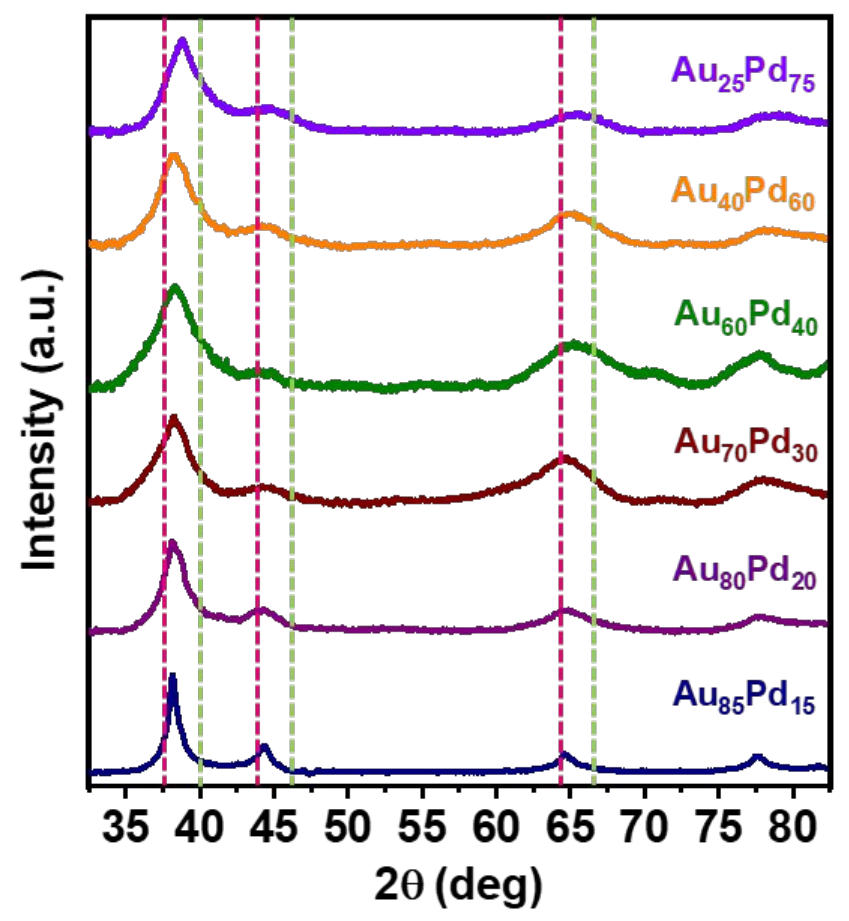

Figure S3: X-ray diffraction patterns for AuPd nanowires of varying composition showing the FCC diffraction peaks for (111), (200), (220) and (311) planes. XRD pattern of Au nanowires is shown in green dashed lines. The standard ICDD pattern for bulk Pd (File \# 00-001-1310) is shown in pink dashed lines. The peaks for AuPd nanowires lie in between these lines indicating alloy nature. 

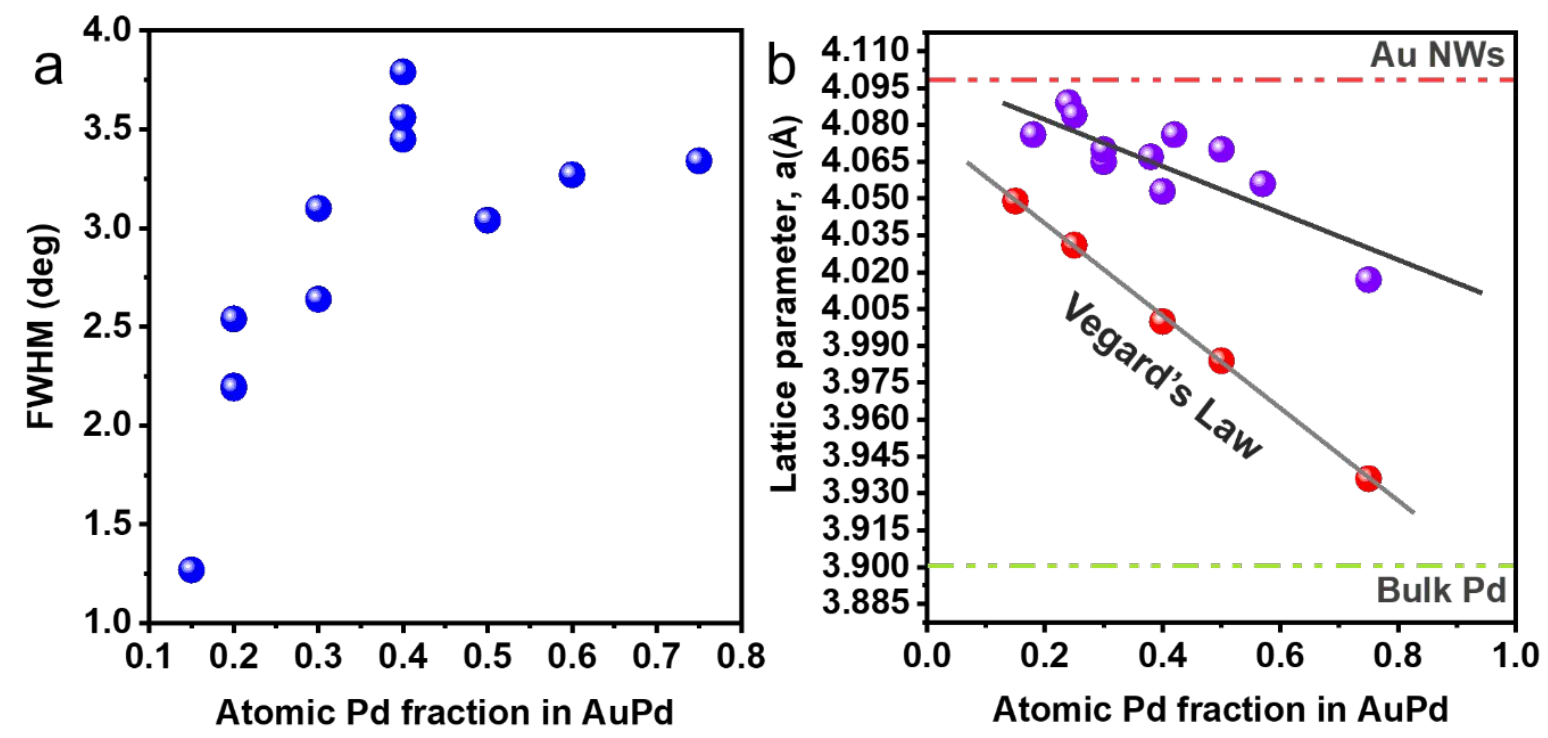

Figure S4: (a) Variation in FWHM of (111) plane with Pd content in compositionally tuned AuPd nanowires. (b) Variation of lattice parameter calculated from d-value of (111) against atomic fraction of Pd in AuPd nanowires. A pseudo linear change in lattice parameter is seen with the increase in Pd content. Red dots in (b) shows the lattice parameter follows the Vegard's law when bulk lattice parameter values are taken from ICSD database. Grey line shows the lattice parameter value of $\mathrm{Au}$ nanowires calculated from (111). Red line shows the lattice parameter value for standard Pd obtained from ICSD database.

XPS Measurements:

The binding energy (BE) value for $\mathrm{Au}_{4 / 2}$ is $83.7 \mathrm{eV}$ for both $\mathrm{Au}_{60} \mathrm{Pd}_{40}$ and $\mathrm{Au}_{50} \mathrm{Pd}_{50}$ compositions. This value is comparable to that of the template Au nanowires under the same XPS measurement conditions4. The $\mathrm{BE}$ value for $\mathrm{Pd} 3 \mathrm{~d}_{5 / 2}$ are $335.1 \mathrm{eV}$ and $335.2 \mathrm{eV}$ for $\mathrm{Pd}_{40}$ and $\mathrm{Pd}_{50}$ compositions of AuPd nanowires. It is interesting to note that the BE shifts are negative for both 
$\mathrm{Au}$ and $\mathrm{Pd}$ in $\mathrm{Au}-\mathrm{Pd}$ alloy system (unlike $\mathrm{Pd}-\mathrm{Cu}$ where $\mathrm{Pd}$ shifts to positive $\mathrm{BE}$ values and $\mathrm{Cu}$ shows the opposite trend) due to charge compensation where Au loses d- electrons and gains spelectrons and Pd vice versa. Interestingly, Au nanowire shows a significant reduction in the BE value for $\mathrm{Au} 4 \mathrm{f}_{7 / 2}$ compared to that of standard bulk Au value $(84.0 \mathrm{eV})$ indicating a high energy surface and a probable alloying with Pd is eminent as Au-Pd is a miscible system. An additional Pd doublet is observed for both compositions at a higher BE with $\mathrm{Pd} 3 \mathrm{~d}_{5 / 2}$ peak settled at $\sim 338 \mathrm{eV}$ which is attributed to Pd-O species. Pd $3 d$ spectrum overlaps with the Au4d $\mathrm{d}_{5 / 2}$. The intensity of $A u 4 d_{5 / 2}$ decreases with the increase in Pd in the sample while the Pd3d peaks become sharper. Such an observation was also made earlier in AuPd supported $\mathrm{TiO} 2$ samples, where it was inferred that an enrichment of Pd leads to sharper Pd3d peaks with lower contribution from $\mathrm{Au} 4 \mathrm{~d}_{5 / 2}$. From the Au4f and Pd3d peak areas, the atomic ratio of $\mathrm{Au}: \mathrm{Pd}$ is calculated using Scofield's relative sensitivity factors $\left(\mathrm{RSF}_{\mathrm{Au} 4 \mathrm{f}}=17.12, \mathrm{RSF}_{\mathrm{Pd} 3 \mathrm{~d}}=16.04\right)$ and the values agree well with WDS measurements (Table S2). 

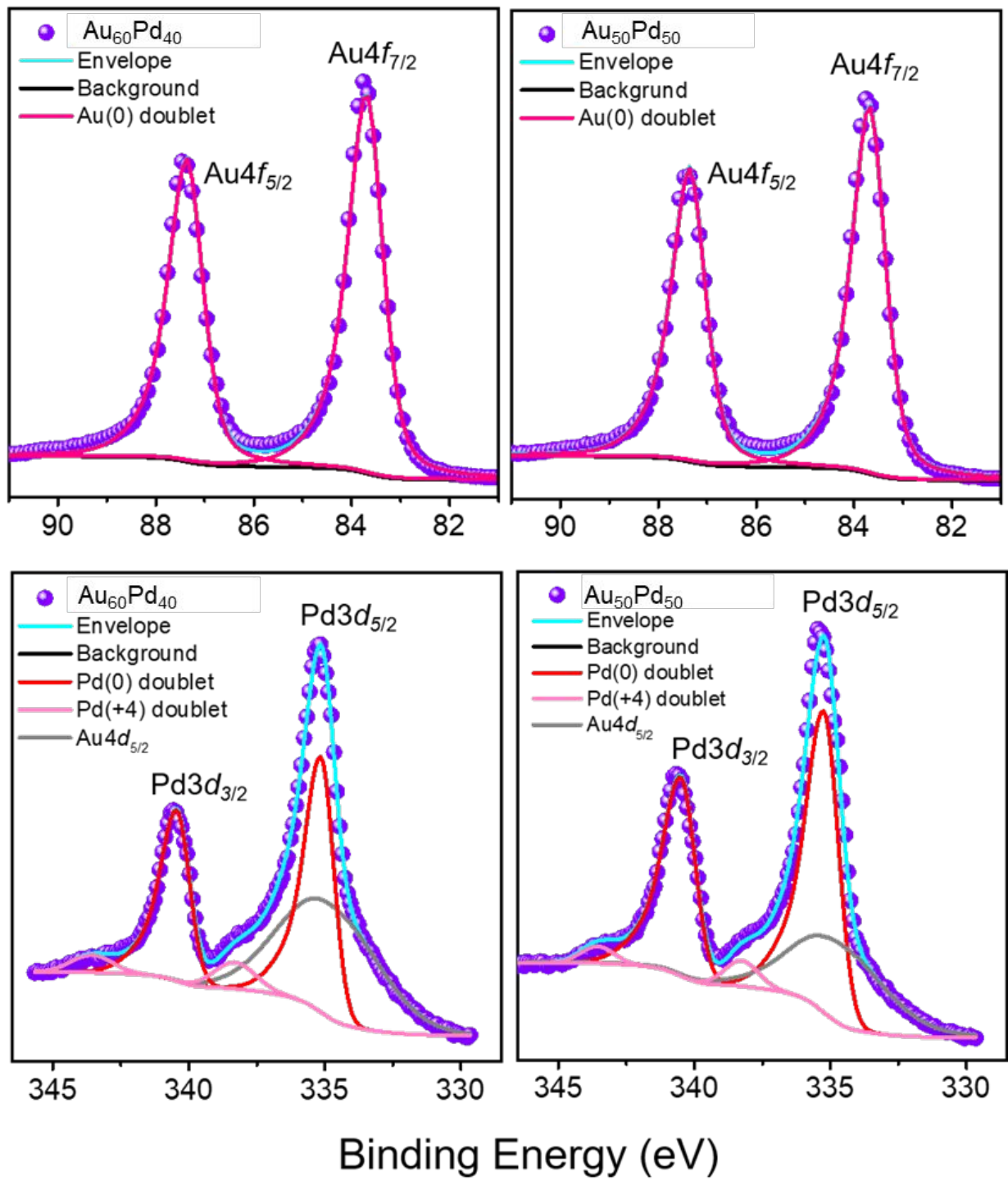

Figure S5: High resolution core level elemental XPS spectrum of Au4f doublet and Pd3d doublet for freshly prepared $\mathrm{Au}_{60} \mathrm{Pd}_{40}$, and $\mathrm{Au}_{50} \mathrm{Pd}_{50}$ compositions. Both $\mathrm{Au}$ and $\mathrm{Pd}$ are metallic in nature. Pd doublet envelope covers the $\mathrm{Au} 4 \mathrm{~d}_{5 / 2}$ peak which helps as a marker to study Pd segregation. 
Table S2: List of values from XPS high resolution core level spectra for AuPd nanowires of varying composition. The calculated atomic fraction of Au and Pd from XPS peak analysis is tabulated at the right-hand side of the table. The binding energy values are compared with NIST database.

\begin{tabular}{|c|c|c|c|c|c|c|}
\hline \multirow{2}{*}{ Sample } & \multirow{2}{*}{ Peak Assigned } & \multirow{2}{*}{ Line type } & \multirow{2}{*}{$\mathrm{BE}(\mathrm{eV})$} & \multirow{2}{*}{ Area } & \multicolumn{2}{|c|}{$\begin{array}{l}\text { Atomic } \\
\text { Fraction }\end{array}$} \\
\hline & & & & & $\mathrm{Au}$ & $\mathrm{Pd}$ \\
\hline \multirow{7}{*}{$\mathrm{Au}_{60} \mathrm{Pd}_{40}$} & \multirow{2}{*}{$\mathrm{Au}(0)$} & $4 f_{7 / 2}$ & 83.7 & 11082 & \multirow{7}{*}{0.63} & \multirow{7}{*}{0.37} \\
\hline & & $4 f_{5 / 2}$ & 87.4 & 8978 & & \\
\hline & \multirow{2}{*}{$\operatorname{Pd}(0)$} & $3 d_{5 / 2}$ & 335.1 & 5759 & & \\
\hline & & $3 d_{3 / 2}$ & 340.4 & 3861 & & \\
\hline & \multirow{2}{*}{$\mathrm{Pd}(+4)$} & $3 d_{5 / 2}$ & 338.3 & 591 & & \\
\hline & & $3 d_{3 / 2}$ & 343.6 & 390 & & \\
\hline & Au4d & $4 d_{5 / 2}$ & 334.9 & 6076 & & \\
\hline \multirow{7}{*}{$\mathrm{Au}_{50} \mathrm{Pd}_{50}$} & \multirow{2}{*}{$\mathrm{Au}(0)$} & $4 f_{7 / 2}$ & 83.7 & 8987 & \multirow{7}{*}{0.48} & \multirow{7}{*}{0.52} \\
\hline & & $4 f_{5 / 2}$ & 87.3 & 7274 & & \\
\hline & \multirow{2}{*}{$\operatorname{Pd}(0)$} & $3 d_{5 / 2}$ & 335.2 & 8997 & & \\
\hline & & $3 d_{3 / 2}$ & 340.0 & 5938 & & \\
\hline & \multirow{2}{*}{$\mathrm{Pd}(+4)$} & $3 d_{5 / 2}$ & 338.2 & 558 & & \\
\hline & & $3 d_{3 / 2}$ & 343.5 & 368 & & \\
\hline & Au4d & $4 d_{5 / 2}$ & 334.7 & 5167 & & \\
\hline
\end{tabular}



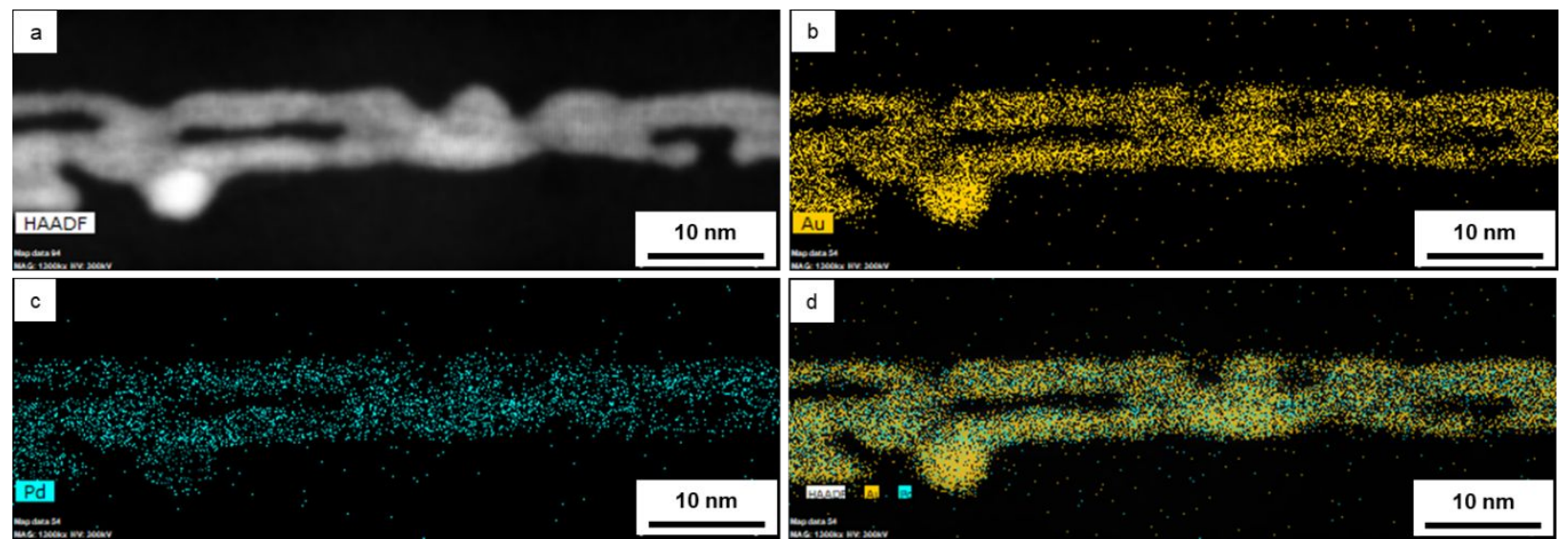

Figure S6: (a), (b), (c), (d) STEM-EDS mapping of $A u_{85} P d_{15}$.
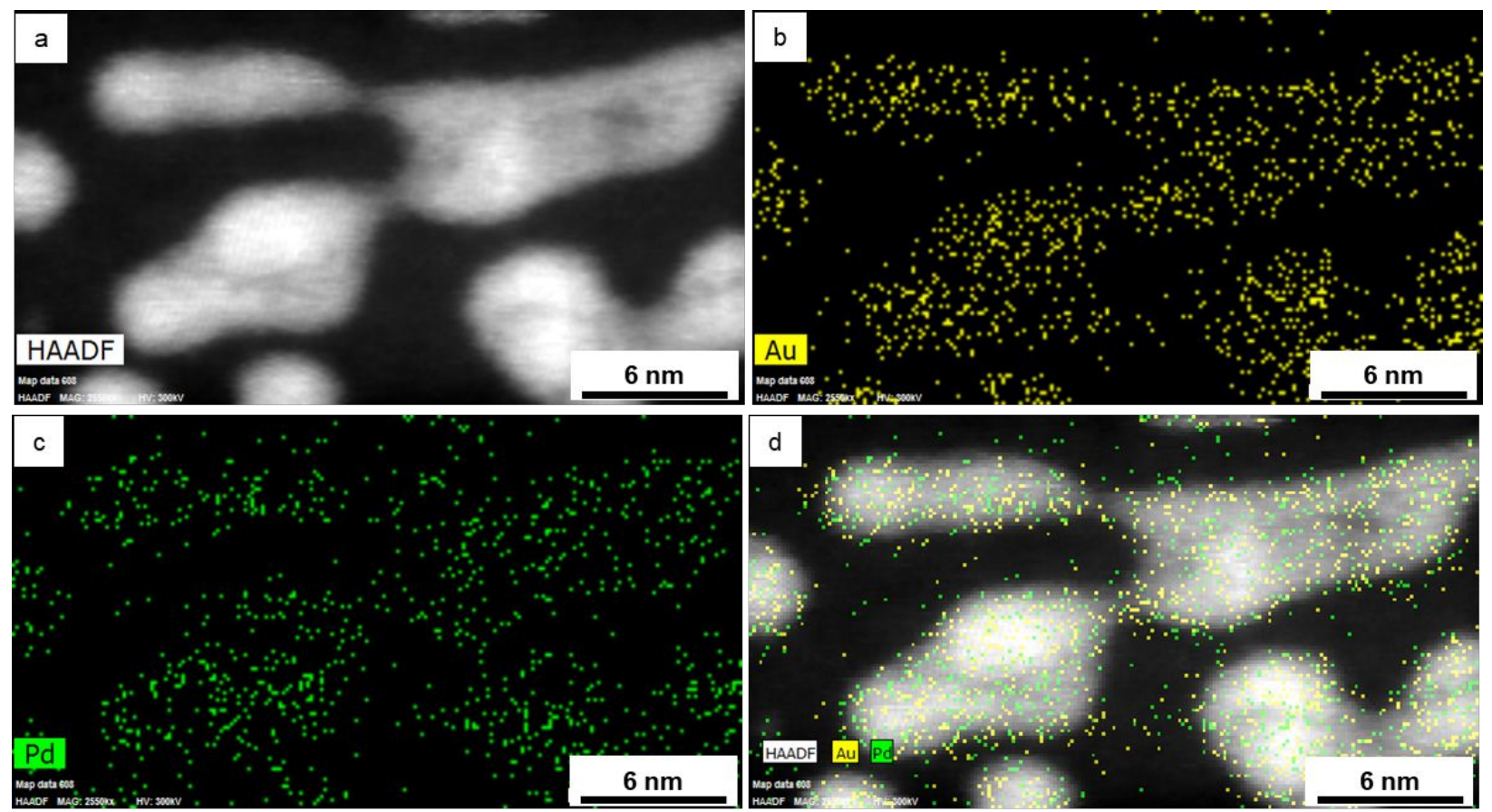

Figure S7: (a), (b), (c), (d) STEM-EDS mapping of $\mathrm{Au}_{75} \mathrm{Pd}_{25}$. 

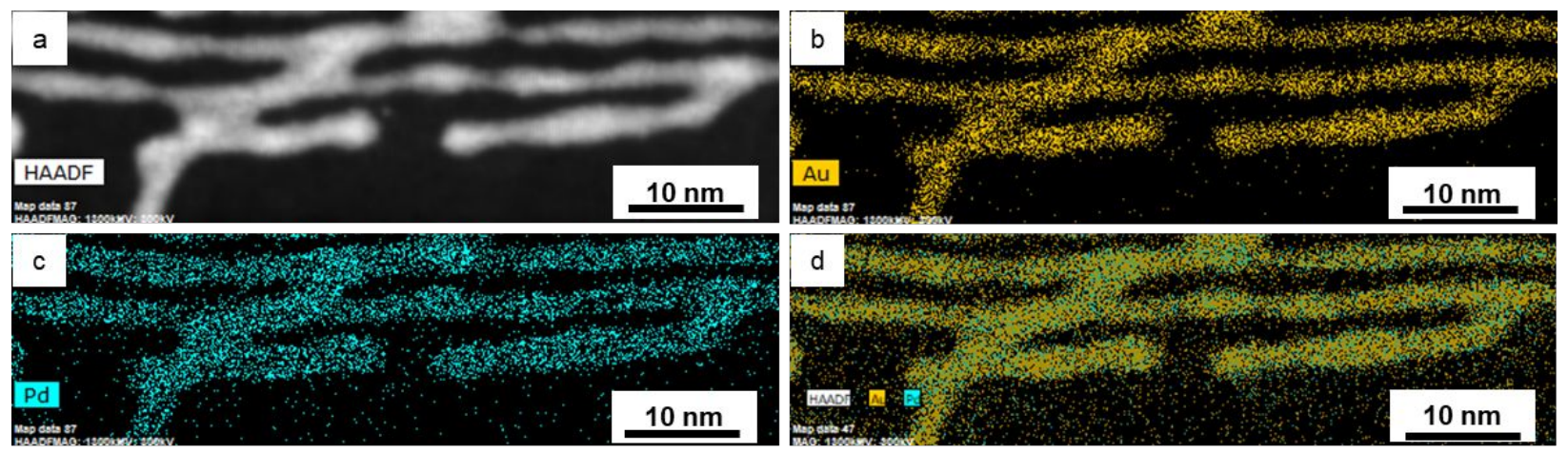

Figure S8: (a), (b), (c), (d) STEM-EDS mapping of $\mathrm{Au}_{60} \mathrm{Pd}_{40}$.
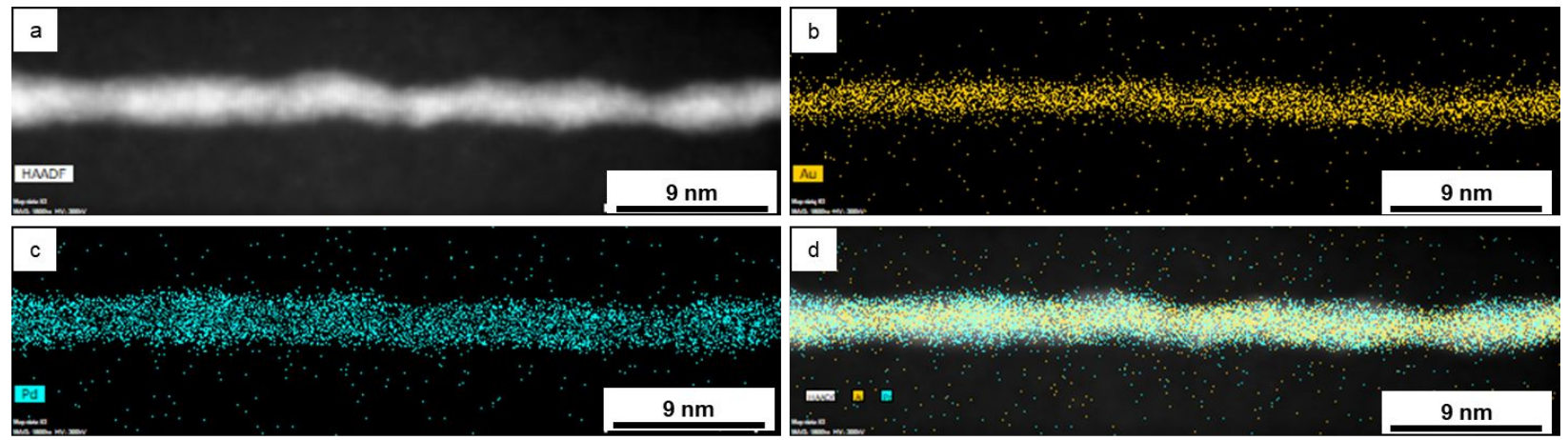

Figure S9: (a), (b), (c), (d) STEM-EDS mapping of $\mathrm{Au}_{50} \mathrm{Pd}_{50}$.
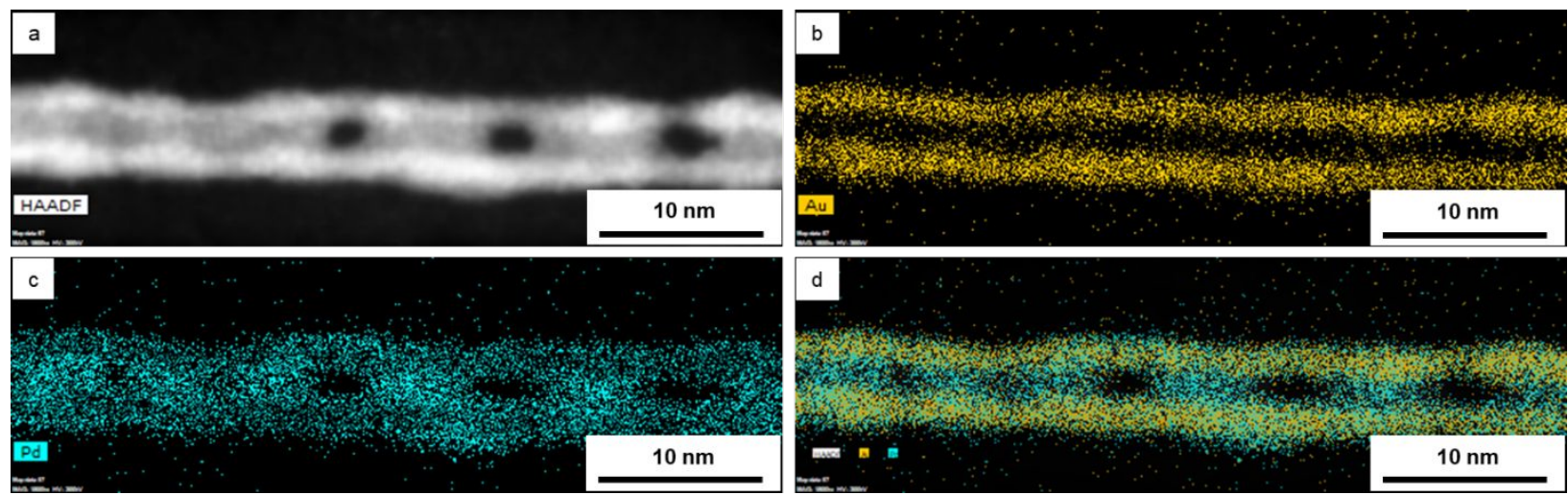

Figure S10: (a), (b), (c), (d) STEM-EDS mapping of $\mathrm{Au}_{75} \mathrm{Pd}_{25}$. 


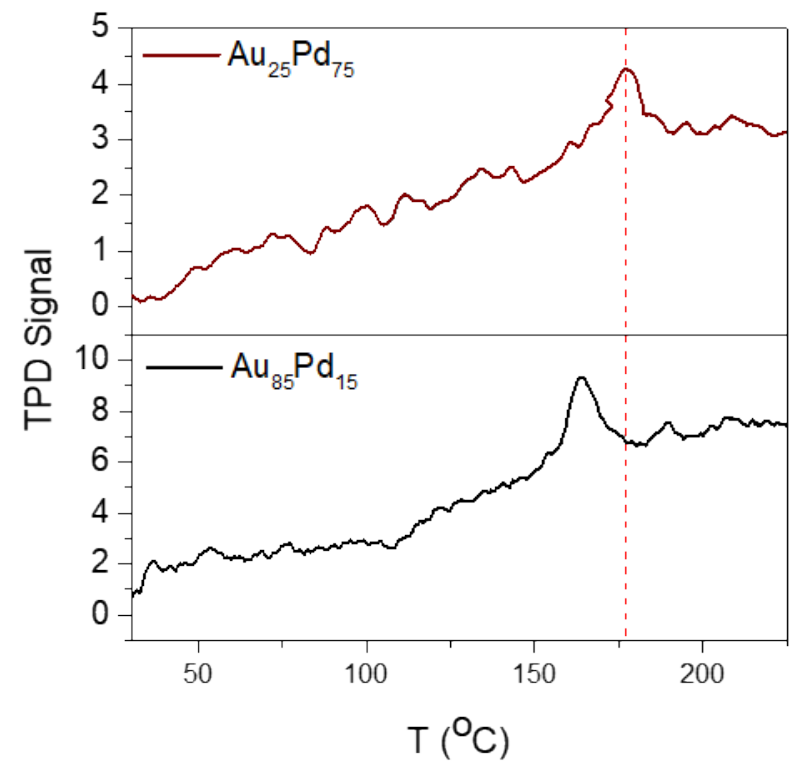

Figure S11: Temperature programmed desorption of $\mathrm{CO}$ from the surface af composition tuned AuPd nanowires. The red line shows the $\mathrm{CO}$ desorption temperature for $\mathrm{Au}_{25} \mathrm{Pd}_{75}$.

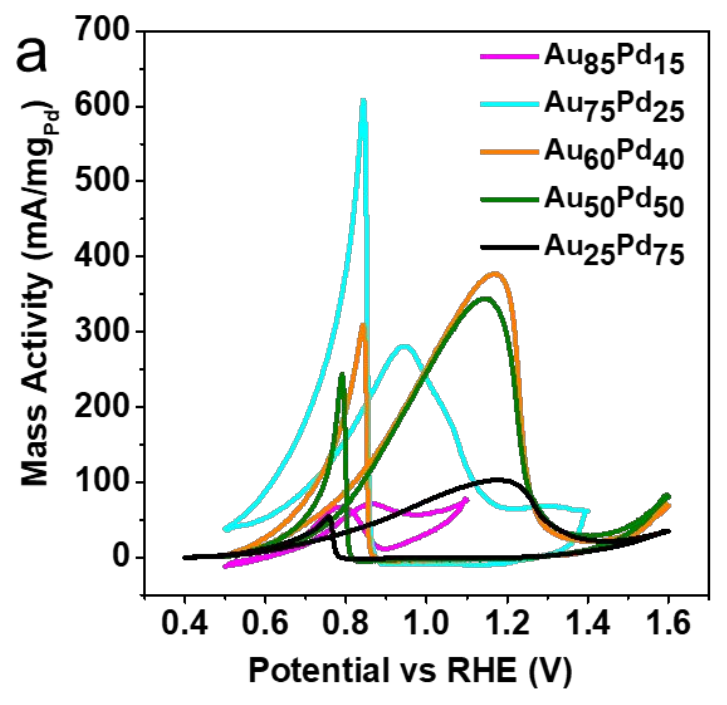


Figure S12: (a) Mass normalized CV scans for AuPd bimetallic nanowires of varying composition in Ar purged $0.5 \mathrm{M} \mathrm{KOH}+1 \mathrm{M} \mathrm{CH}_{3} \mathrm{OH}$ solution.

Table S3: The values for peak MOR potential and for the ratio of forward scan peak current to that of backward scan are listed for composition tuned

\begin{tabular}{|c|c|c|}
\hline & \multicolumn{2}{|c|}{ Composition tuned } \\
\cline { 2 - 3 } & $\begin{array}{c}\text { MOR } \\
\text { Potential }(\mathrm{V})\end{array}$ & $\mathrm{I}_{f} / I_{b}$ \\
\hline $\mathrm{Au}_{85} \mathrm{Pd}_{15}$ & 0.86 & 1.06 \\
\hline $\mathrm{Au}_{75} \mathrm{Pd}_{25}$ & 0.94 & 0.46 \\
\hline $\mathrm{Au}_{60} \mathrm{Pd}_{40}$ & 1.16 & 1.22 \\
\hline $\mathrm{Au}_{50} \mathrm{Pd}_{50}$ & 1.17 & 1.40 \\
\hline $\mathrm{Au}_{25} \mathrm{Pd}_{75}$ & 1.20 & 1.92 \\
\hline
\end{tabular}



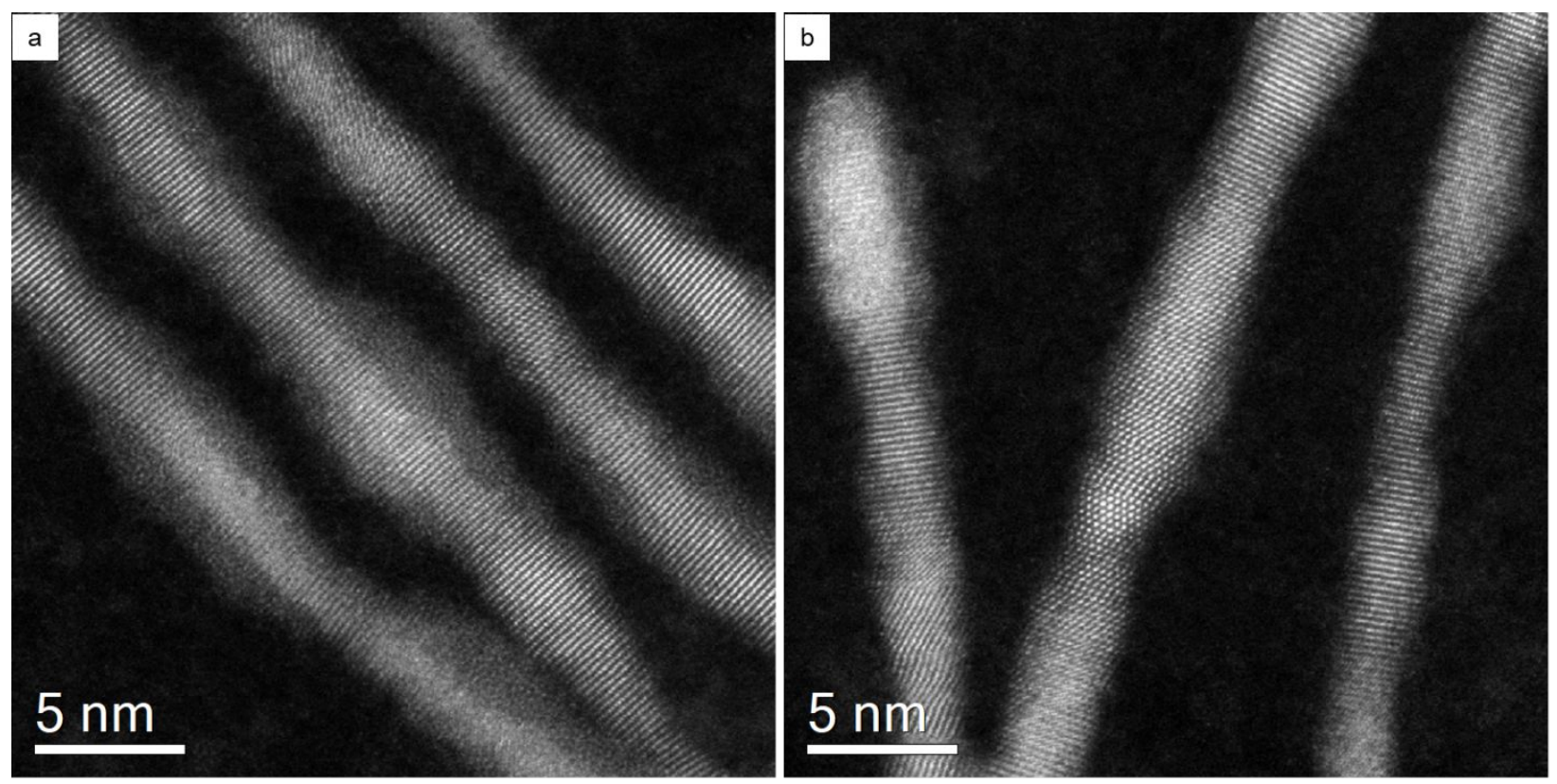

Figure S13: (a), (b) Z-contrast HAADF-STEM images of $\mathrm{CO}$-annealed $\mathrm{Au}_{50} \mathrm{Pd}_{50}$ bimetallic nanowire.

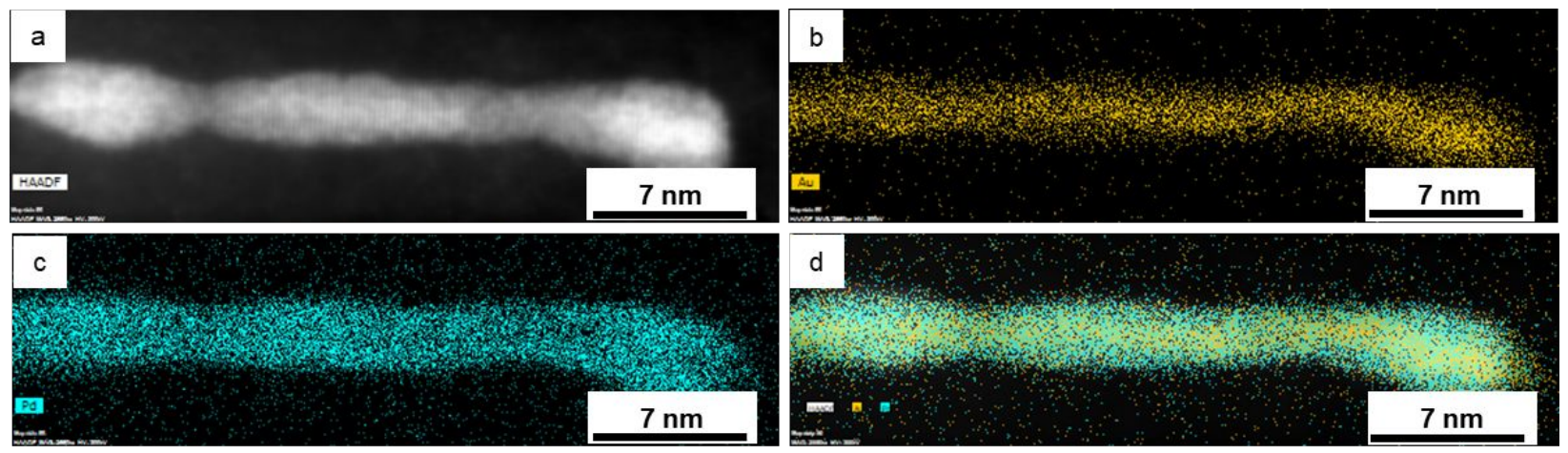

Figure S14: (a), (b), (c), (d) STEM-EDS mapping of CO-annealed $\mathrm{Au}_{60} \mathrm{Pd}_{40}$. 


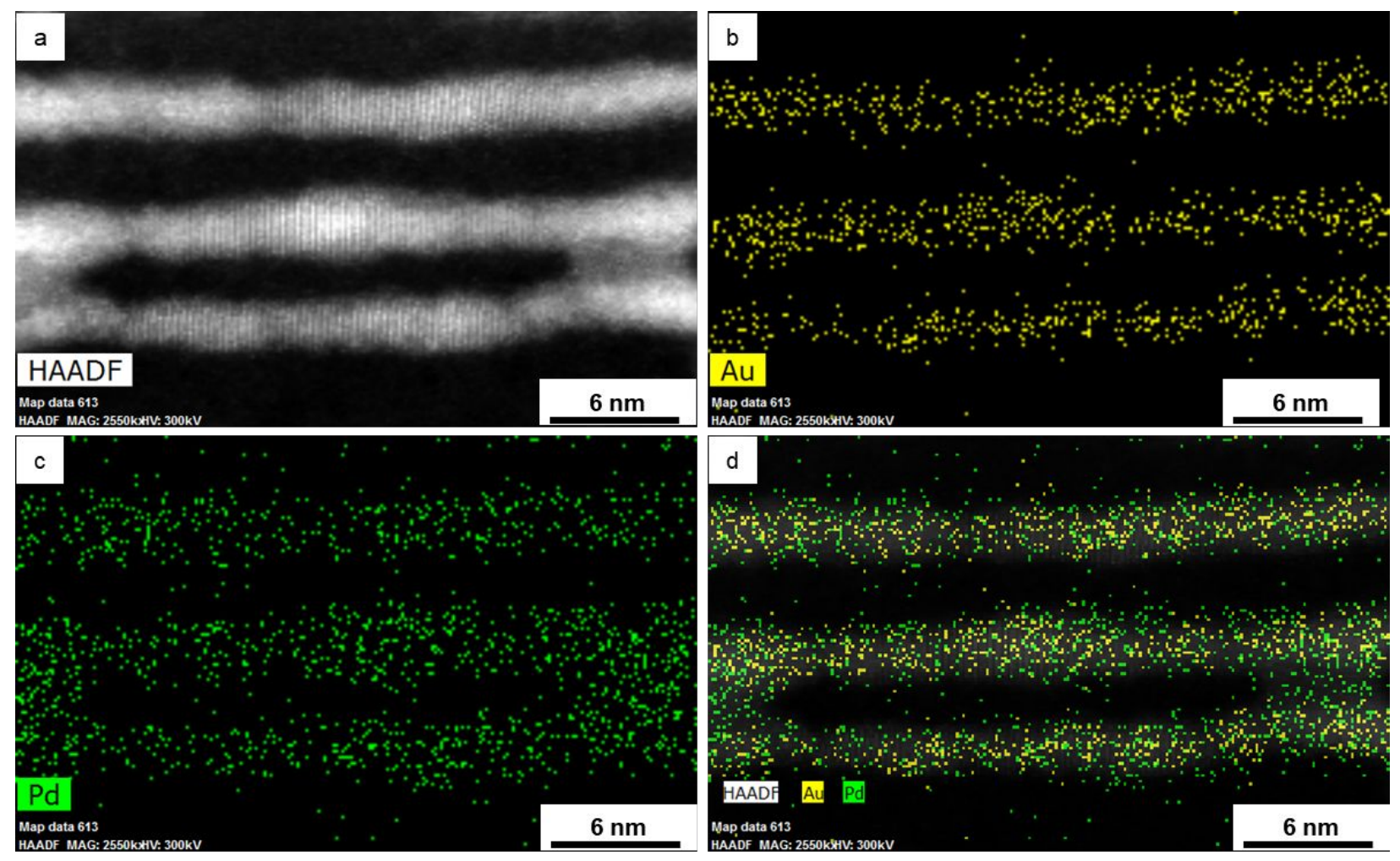

Figure S15: (a), (b), (c), (d) STEM-EDS mapping of CO-annealed $\mathrm{Au}_{50} \mathrm{Pd}_{50}$. 


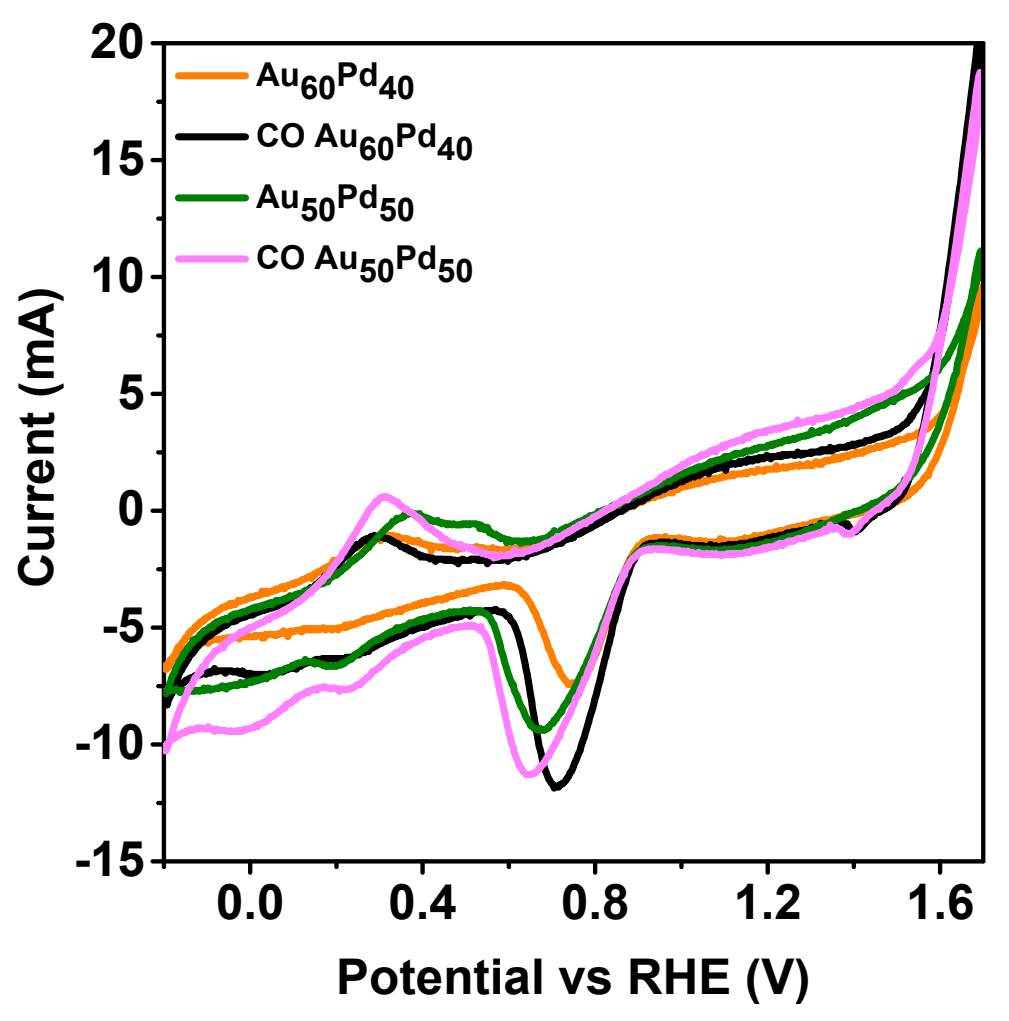

Figure S16: CV scans for as synthesized and Pd segregated (CO annealed) $\mathrm{Au}_{60} \mathrm{Pd}_{40}$ and $\mathrm{Au}_{50} \mathrm{Pd}_{50}$ in Ar purged 0.5 M KOH solution.

Table S4: The values for peak MOR potential and for the ratio of forward scan peak current to that of backward scan are listed for CO segregated AuPd samples in the above table. 


\begin{tabular}{|c|c|c|}
\hline \multirow{2}{*}{ Samples } & \multicolumn{2}{|c|}{ CO segregated } \\
\cline { 2 - 3 } & $\begin{array}{c}\text { MOR } \\
\text { Potential }(\mathrm{V})\end{array}$ & $\mathrm{I}_{f} / \mathrm{I}_{b}$ \\
\hline $\mathrm{Au}_{60} \mathrm{Pd}_{40}$ & 1.11 & 0.84 \\
\hline $\mathrm{Au}_{50} \mathrm{Pd}_{50}$ & 1.03 & 1.08 \\
\hline
\end{tabular}

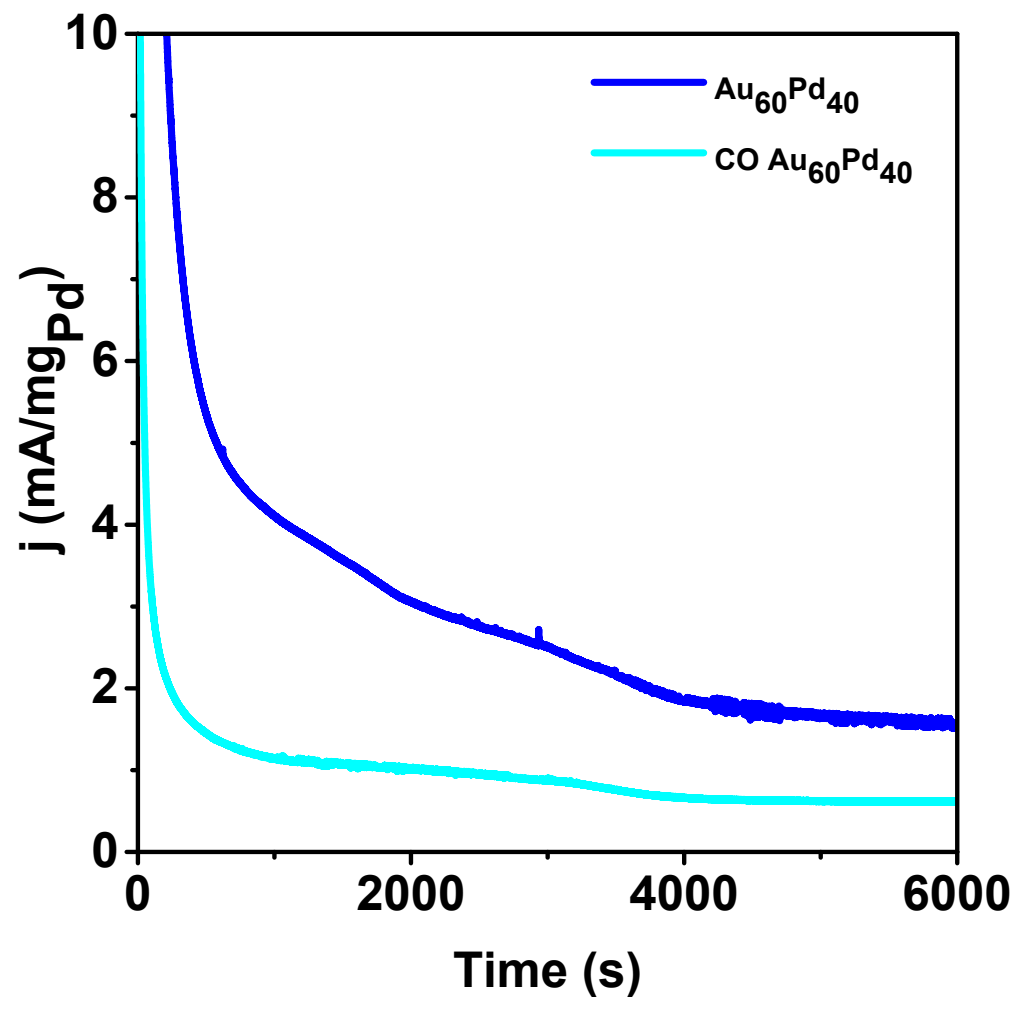

Figure S17: Chronoamperometry of $\mathrm{CO}$ annealed $\mathrm{Au}_{60} \mathrm{Pd}_{40}$ and $\mathrm{Au}_{60} \mathrm{Pd}_{40}$ in $\mathrm{Ar}$ purged $0.5 \mathrm{M} \mathrm{KOH}$ solution. 


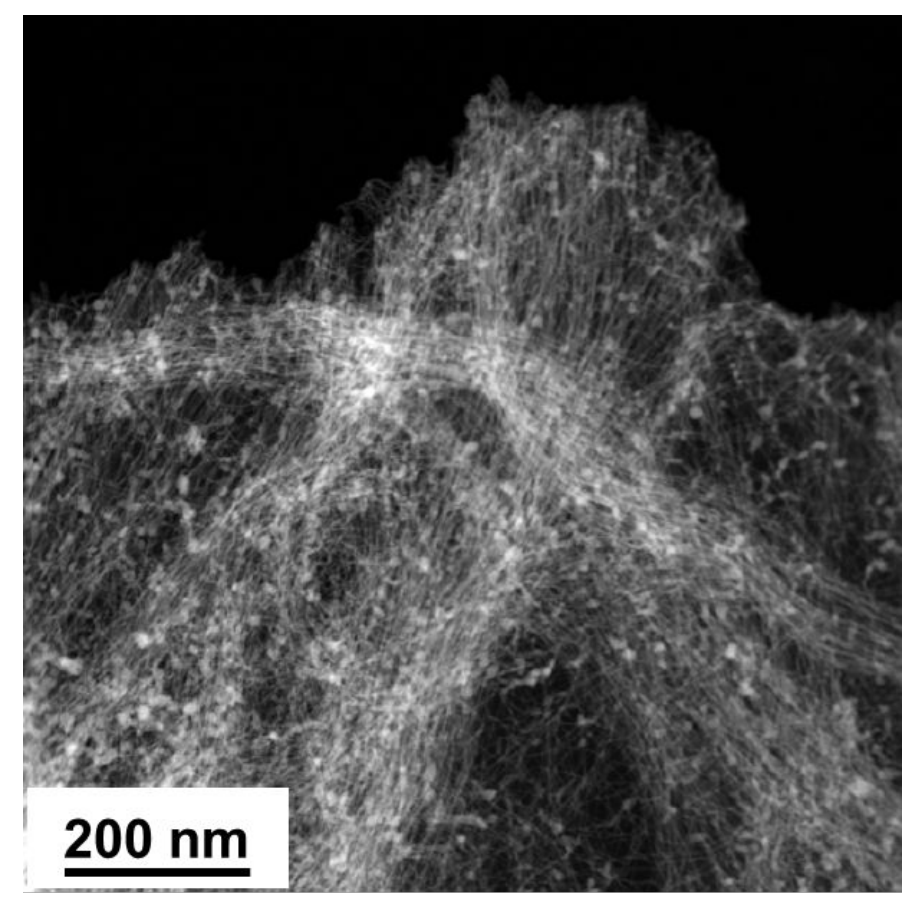

Figure S18: HAADF-STEM image of $\mathrm{Au}_{75} \mathrm{Pd}_{25}$ after catalysis.

Table S5: Comparison of previously reported materials with present work

\begin{tabular}{|c|c|c|c|c|}
\hline Electrocatalyst & Solution & Onset Potential & Current Density & References \\
\hline AuPd-4 & $\begin{array}{c}1 \mathrm{M} \mathrm{NaOH}+1 \\
\mathrm{M} \text { methanol }\end{array}$ & $-0.323 \mathrm{~V}$ & $1.27 \mathrm{mAcm}^{-2}$ & 3 \\
\hline AuPd-5 & $\begin{array}{c}1 \mathrm{M} \mathrm{NaOH}+1 \\
\mathrm{M} \text { methanol }\end{array}$ & $-0.385 \mathrm{~V}$ & $1.37 \mathrm{mAcm}^{-2}$ & 3 \\
\hline $\begin{array}{c}\text { AuPd@Pd NC } \\
\text { s/N-RGOH }\end{array}$ & $\begin{array}{l}0.5 \mathrm{M} \mathrm{KOH}+ \\
1.0 \mathrm{M} \text { methanol }\end{array}$ & $0.859 \mathrm{~V}$ & $0.98 \mathrm{~mA} \mathrm{~cm}^{-2}$ & 4 \\
\hline $\mathrm{Au}_{60} \mathrm{Pd}_{40}$ & $\begin{array}{c}0.5 \mathrm{M} \mathrm{KOH}+1 \\
\mathrm{M} \text { methanol }\end{array}$ & $1.16 \mathrm{~V}$ & $2.15 \mathrm{~mA} \mathrm{~cm}^{-2}$ & This work \\
\hline
\end{tabular}


All the first principle calculations are performed using Density functional theory (DFT) as implemented in the Vienna ab initio simulation package (VASP) ${ }^{5}$. Electron projector augmented wave $(\mathrm{PAW})^{6}$ pseudopotentials describe the electron-ion interactions and the electronic exchange and correlations were approximated by a Perdew-Burke-Ernzerhof $(\mathrm{PBE})^{7}$ generalized gradient approximation (GGA) including spin polarization effects. The periodic images were separated by a $15 \AA ̊$ vacuum along z-direction to prevent spurious interactions. The Brillouin zone was sampled by a 3x5x1 and 2x3x1 Monkhorst-Pack grid for AuPd(110) and both the core@ shell (110) surfaces (AuPd@Pd and AuPd@Au) structure optimization, respectively. All structures were fully relaxed using a conjugate gradient scheme until the energies and each component of forces were less than 10-6 eV and $0.01 \mathrm{eV} \AA-1$, respectively.

The free energy change for each intermediate is calculated using the computational hydrogen electrode model $(\mathrm{CHE})^{8}$ as follows:

$\Delta \mathrm{G}=\Delta \mathrm{E}_{\mathrm{ads}}+\Delta \mathrm{ZPE}-\mathrm{T} \Delta \mathrm{S}-\mathrm{eU}$,

Where, $\Delta \mathrm{E}_{\mathrm{ads}}, \Delta \mathrm{ZPE}$, and $\Delta \mathrm{S}$ are the adsorption energy, the zero point energy, and the entropic contributions (vibrational degree of freedom only) for the adsorbed species, respectively. e and $U$ are the number of electrons transferred and the applied electrode potential with respect to the reversible hydrogen electrode (RHE).

The limiting potential (UL) for a reaction is calculated following the equation given below:

$$
U L=\frac{-\Delta G \max }{e}
$$


Where, $-\Delta G \max$ is the difference between the free energies of adsorption for the potential limiting step (PLS).

Methanol Oxidation pathway 

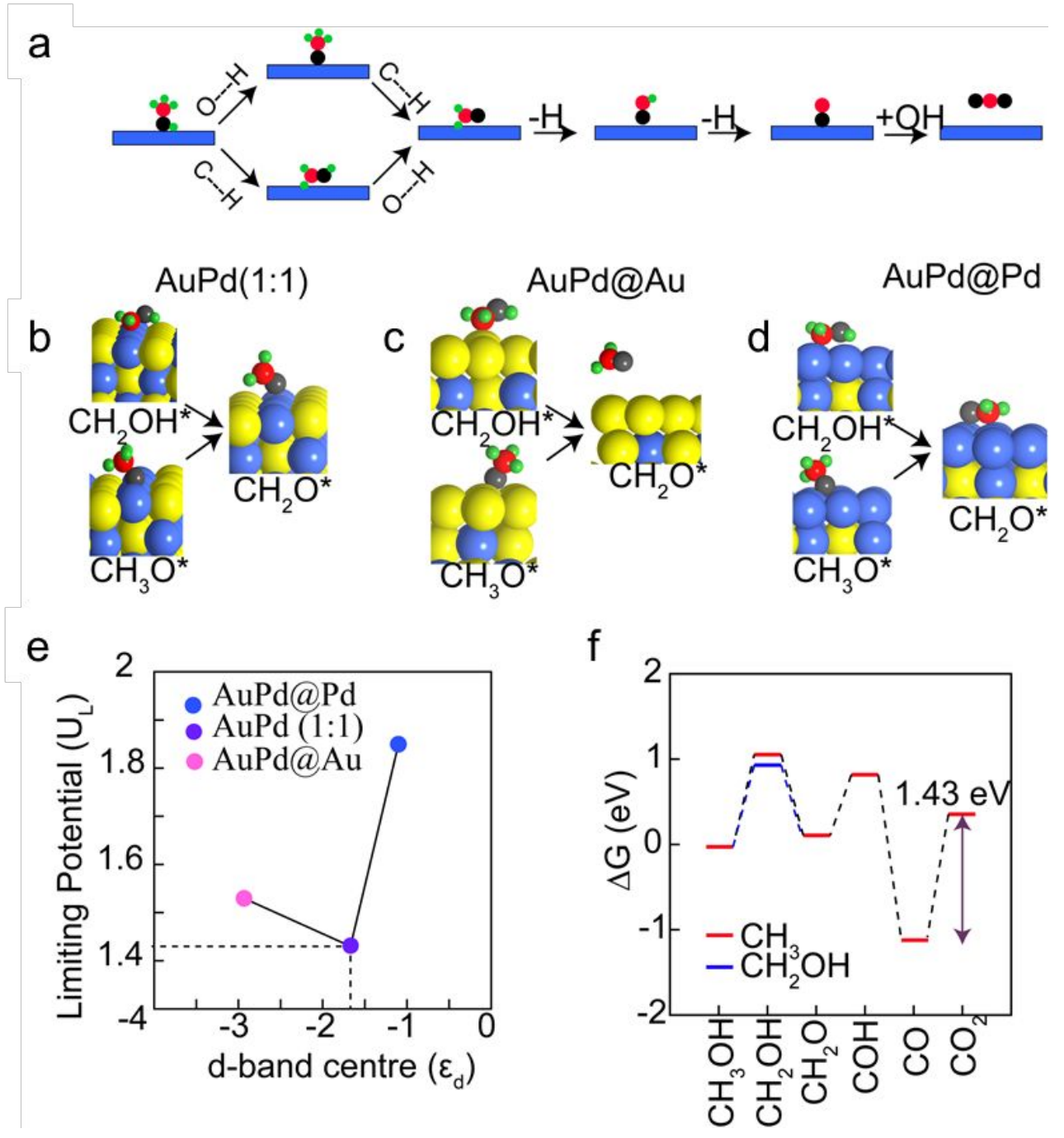

Figure S19. (a) Methanol oxidation pathways on the surface of catalyst. Black, red, green, yellow, and blue balls represent oxygen, carbon, hydrogen, gold, and palladium, respectively. (b-d) The optimized geometry of the intermediates involved in two pathways for the three systems AuPd(1:1), AuPd@Pd and AuPd@Au. (e)Limiting potential (UL) for elementary steps in MOR mechanism as a function of d-band center. (f) Reaction coordinate for the methanol oxidation reaction on $\operatorname{AuPd}(1: 1)$ surface. The two pathways involved are represented in blue and red. The rate limiting step is oxidation of $\mathrm{CO}$ to $\mathrm{CO} 2$ and limiting potential is $1.43 \mathrm{eV}$. Horizontal dashed line represents the limiting potential. 
The methanol oxidation can follow two pathways one by $\mathrm{O}-\mathrm{H}$ bond scission and other by $\mathrm{C}-\mathrm{H}$ bond scission leading to $\mathrm{CH}_{3} \mathrm{O}$ and $\mathrm{CH}_{2} \mathrm{OH}$ respectively. Both of these intermediates lead to $\mathrm{CH}_{2} \mathrm{O}$ (Figure S17a-c). When the $\mathrm{CH}_{2} \mathrm{OH}$ is more favorable the $\mathrm{CH}_{2} \mathrm{O}$ intermediate is bound to the surface through both $\mathrm{C}$ and $\mathrm{O}$. For the case of Pd over the surface $\mathrm{CH}_{2} \mathrm{OH}$ is more stable (Figure S17d) compared to $\mathrm{CH}_{3} \mathrm{O}$ (binds through oxygen) by $\sim 0.4 \mathrm{eV}$, which makes it more feasible to get converted to $\mathrm{CH}_{2} \mathrm{O}$ attached through $\mathrm{C}$ and $\mathrm{O}$. While for the case of $\mathrm{AuPd}(1: 1)$ the difference is not that significant $(\sim 0.04 \mathrm{eV})$ it can go through any of the paths which makes the $\mathrm{CH}_{2} \mathrm{O}$ intermediate bind only through C. Figure S17e suggests that for AuPd (1:1) surface, the limiting potential is almost $\sim 1.4 \mathrm{~V}$ whereas for the Pd rich surface the limiting potential is $\sim 1.85 \mathrm{~V}$ and for the Au-rich case the $\mathrm{CH}_{2} \mathrm{O}$ intermediate has very low binding to the surface such that its conversion to $\mathrm{COH}^{*}$ is difficult and it can completely get released as formaldehyde(HCHO). Hence, the limiting step in the conversion of methanol to $\mathrm{CO}_{2}$ on Au rich surface is $\mathrm{CH}_{2} \mathrm{O}$ to $\mathrm{COH}^{*}$ and the limiting potential is $\sim 1.57 \mathrm{~V}$. These results align well with the experimental results where it was found that as the amount of Pd increases the potential increases and for the Au rich case the reaction does not reach up to $\mathrm{CO}_{2}$ it gives $\mathrm{HCHO}$ as a product at lower potentials. The reason for this can be analyzed through the d-band center as shown in Figure S17e, the limiting potential increases as 
the d-band center moves towards the Fermi-level. With the d-band center nearer to the Fermi-level

the $\mathrm{CO}^{*}$ binding is very strong which leads to $\mathrm{CO}$ - poisoning over the surface. As we move away

from the Fermi-level the binding become extremely weak such that $\mathrm{CH}_{2} \mathrm{O}$ itself get released from

the surface restricting further oxidation, whereas for the case of $\operatorname{AuPd}(1: 1)$ the d-band center lies

at an optimum position such that the binding of $\mathrm{CO}^{*}$ is neither too strong resulting in $\mathrm{CO}-$

poisoning nor too weak so that it cannot bind the intermediates for further oxidation. The

thermodynamics of the methanol oxidation on $\operatorname{AuPd}(1: 1)$ is shown in Figure S17f. It is clear from

the figure that from all the steps involved in the reaction the potential limiting step (PLS) is found

to be the oxidation of $\mathrm{CO}^{*}$ to $\mathrm{CO}_{2}$ on $\operatorname{AuPd}(1: 1)$ with a limiting potential of $\sim 1.4 \mathrm{~V}$, which is

lowest amongst all the selected surfaces.

\section{References:}

(1) Feng, H.; Yang, Y.; You, Y.; Li, G.; Guo, J.; Yu, T.; Shen, Z.; Wu, T.; Xing, B. Simple and Rapid Synthesis of Ultrathin Gold Nanowires, Their Self-Assembly and Application in Surface-Enhanced Raman Scattering. Chemical Communications 2009, No. 15, 1984 1986.

(2) Chatterjee, D.; Shetty, S.; Müller-Caspary, K.; Grieb, T.; Krause, F. F.; Schowalter, M.; Rosenauer, A.; Ravishankar, N. Ultrathin Au-Alloy Nanowires at the Liquid-Liquid Interface. Nano Letters 2018, 18 (3), 1903-1907.

(3) Luo, L. M.; Zhan, W.; Zhang, R. H.; Chen, D.; Hu, Q. Y.; Guo, Y. F.; Zhou, X. W. 
Ternary CoAuPd and Binary AuPd Electrocatalysts for Methanol Oxidation and Oxygen Reduction Reaction: Enhanced Catalytic Performance by Surface Reconstruction. Journal of Power Sources 2019, 412, 142-152.

(4) Yu, D. X.; Wang, A. J.; He, L. L.; Yuan, J.; Wu, L.; Chen, J. R.; Feng, J. J. Facile Synthesis of Uniform AuPd@Pd Nanocrystals Supported on Three-Dimensional Porous N-Doped Reduced Graphene Oxide Hydrogels as Highly Active Catalyst for Methanol Oxidation Reaction. Electrochimica Acta 2016, 213, 565-573.

(5) Kresse, G.; Furthmüller, J. Efficiency of Ab-Initio Total Energy Calculations for Metals and Semiconductors Using a Plane-Wave Basis Set. Computational Materials Science 1996, 6 (1), 15-50.

(6) Joubert, D. From Ultrasoft Pseudopotentials to the Projector Augmented-Wave Method. Physical Review B - Condensed Matter and Materials Physics 1999, 59 (3), 1758-1775.

(7) Perdew, J. P.; Burke, K.; Ernzerhof, M. Generalized Gradient Approximation Made Simple. Physical Review Letters 1996, 77 (18), 3865-3868.

(8) Peterson, A. A.; Abild-Pedersen, F.; Studt, F.; Rossmeisl, J.; Nørskov, J. K. How Copper Catalyzes the Electroreduction of Carbon Dioxide into Hydrocarbon Fuels. Energy and Environmental Science 2010, 3 (9), 1311-1315. 